\title{
The Equation of Number the Total Internal of Reflection Angles Occur in Optical Fiber
}

\author{
Zaid A. Hasan \\ Department of physics, College of Education for Pure Science, Babylon University, Iraq
}

\begin{abstract}
This studding is deduced a mathematical model is determined the number of total internal reflection angles occur in optical fiber is depend on the many parameters (optical fiber length, diameter a core, reflection angle). The number of total internal angles is direct proportionality with optical fiber length while is indirect proportionality with optical fiber diameter a core and the tan function for reflection angle. The mathematical model is useful at design the optical fiber for less the attenuation.
\end{abstract}

Keywords: optical fiber, total internal reflection

\section{Introduction and Basic Concept}

In $1840_{\mathrm{s}}$ Swiss physicist Daniel Collodon and French physicist Jacques Babinet showed that light could beguided along jets of water for fountain display. British physicist John Tyndall popularized light guiding in a demonstration he first used in 1854, guiding light in jet of water flowing from a tank. By the turn of the century, inventors realized that bent quartz rods could carry light, and patented them as dental illuminators. By the $1940_{\mathrm{s}}$, many doctors used illuminated plexiglass tongue depressors. Optical fibers went a step further. they are essentially transparent rods of glass or plastic stretched so they are long and flexible.Duringthe $1920_{\mathrm{s}}$, John Logie Baird in England and Clarence W .Hansell in the United States patented the idea of using arrays of hollow pipes or transparent rods to transmit images for television or facsimile systems .However, the first person known to have demonstrated image transmission through a bundle of optical fibers was Heinrich Lamm, than a medical student in Munich. His goal was to look inside inaccessible parts of the body, and in a 1930 paper he reported transmitting the image of a light bulb filament through a short bundle.However, the unclad fibers transmitted images poorly, and the rise of the Nazis forced Lamm, a Jew, to move to America and abandon his dreams of becoming a professor of medicine [1].In 1951, Holger Moller Hansen applied for a Danish patent on fiber-optic imaging.However, the Danish patent office denied his application, citing the Baird and Hansell patent, and Moller Hansen was unable to interest companies in his invention. Nothing more was reported on fiber bundles until 1954, when Abraham van Heel of the Technical University of Delft in Holland and Harold.H.Hopkins and NarinderKapany of Imperial Collage in London separately announced imaging bundle in the prestigious British journal. Neither van Heel nor Hopkins and kapany made bundles that could carry light far, but their reports the fiber optics revolution. The crucial innovation was made by van Heel, stimulated by a conversation with the American optical physicist Brian O'Brien.All earlier fibers were "bare", with total internal reflection at a glass - air interface. van Heel covered a bare fiber or glass or plastic with a transparent cladding of lower refractive index .This protected the total-reflection surface from contamination, and greatly reduced crosstalk between fibers .The next key step was development of glass -clad fibers, by Lawrence Curtiss, then an undergraduate at the University of Michigan working part-time on a project to develop an endoscope to examine the inside of the stomach with physician Basil Hirschowitz, physicist C.Wilbur Peter. By 1960, glass -clad fibers had attenuation of about one decibel per meter, fine for medical imaging, but much too high for communication [2].

Optical fibers had attracted some attention because they were analogous in theory to plastic dielectric waveguides used certain microwave applications .In 1961,Elias Snitzer at American Optical, working with Hicks at Mosaic Fabrications (now Galileo Electro-Optics),demonstrated the similarity by drawing fibers with cores so small they carried light in only one waveguide mode. However virtually everyone considered fibers too lossy for communications; attenuation of decibel per meter was fine for looking inside the body, but communications operated over much longer distances, and required loss no more than 10 or 20 decibel per kilometer [3]. In 1970, the first low -loss optical fiber was developed. The optical fiber, made from silica glass about 250 micrometers in diameter - about the size of human hair -was used to propagate light in a lab environment. This was the beginning of fiber optics. Shortly after this, the process of manufacturing thin glass fiber strands was perfected and, in the mid $1970_{\mathrm{s}}$, Corning Inc.made fiber optic cable available commercially [4].Attenuations in modern optical cables are far less than those in electrical copper cables, leading to long-haul fiber connections with repeater distances of 70-150 kilometer (43$93 \mathrm{mi}$ ). The erbium-doped fiber amplifier, which reduced the cost of long- distance fiber systems by reducing or even in many cases eliminating the need for optical-electrical-optical repeaters, was co-developed by teams led by David N.Payne of University of Southampton, and Emmanuel Desurvire at Bell Lab in 1986 . The more robust optical fiber commonly used today utilizes glass for both core and sheath and is therefore less prone to aging processes. It was invented by Gerhard Bernsee in 1973 of Schott Glass in Germany. In 1991, the emerging field of photonic crystals led to the 


\section{International Journal of Science and Research (IJSR) \\ ISSN (Online): 2319-7064 \\ Index Copernicus Value (2013): 6.14 | Impact Factor (2014): 5.611}

development of photonic crystal fiber which guides light by means of diffraction from a periodic structure, rather than total internal reflection. The first photonic crystal fiber became commercially available in 2000 . Photonic crystal fibers can be designed to carry higher power than conventional fiber, and their wavelength dependent properties can be manipulated to improve their performance in certain applications [5]. The optical fiber is manufacturing from many materials such as silica, fluorides , plastic ( polymer ) or phosphates [ 6], use in many fields [communication, telephone, video, television, images, computers, local area networks $\left(\mathrm{LAN}_{\mathrm{S}}\right)$, wide area networks $\left(\mathrm{WAN}_{\mathrm{S}}\right)$, and control systems]

\subsection{Principle of Operation}

An optical fiber is a cylindrical dielectric waveguide (nonconducting waveguide) that transmits light along its axis, by the process of total internal reflection. The fiber consists of a core surrounded by a cladding layer, both of which are made of dielectric material. To confine the optical signal in the core, the refractive index of the core must be greater than that of the cladding. The boundary between the core and cladding may either be abrupt, in step-index fiber, or gradual, in graded index fiber. [7].

When light traveling in dense medium hits a boundary at a steep angle (larger than the "critical angle" for the boundary), the light will be completely reflected. This effect is used in optical fibers to confine light in the core. Light travels along the fiber bouncing back and forth off of the boundary. Because the light must strike the boundary with an angle greater than the critical angle, only light that enters the fiber within a certain range of angles can travel down the fiber without leaking out .This range of angles is called the acceptance cone of the fiber. The size of this acceptance cone is a function of the refractive index difference between the fiber's core and cladding. In simpler terms, there is a maximum angle from the fiber axis at which light may enter the fiber so that it will propagate, or travel, in the core of the fiber. The sine of this maximum angle is the numerical aperture $\left(\mathrm{N}_{\mathrm{A}}\right)$ of the fiber .Fiber with a larger $\mathrm{N}_{\mathrm{A}}$ requires less precision to splice and work with than fiber with a smaller $\mathrm{N}_{\mathrm{A}}$. Single mode fiber has

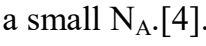

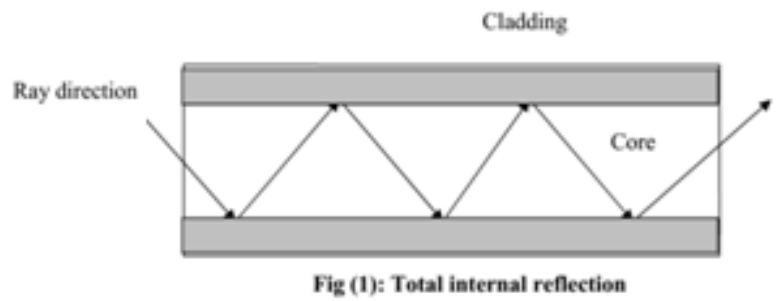

\subsection{Optical Fiber Composition and Types}

An optical fiber is a long, cylindrical, transparent material that confines and propagates light waves (see Fig 2). It is comprised of three layers, the center core that carries the light, the cladding layer that covers the core which confines the light to the core, and the coating that provides protection for the cladding. The core and cladding are commonly made from pure silica glass $\left(\mathrm{SiO}_{2}\right)$, while the coating is a plastic or acrylate cover . It is interesting to note just how transparent the glass in the core of an optical fiber The picture window you have at home in your living room is $(1 / 8)$ in thick plate glass . The core and cladding layers differ slightly from each other in their composition due to small quantities of material such as boron or germanium added during the manufacturing process. This alters the index of refraction characteristic of both layers, resulting in the light confinement properties needed to propagate the light rays. The index of refraction in the silica core is approximately 1.5 and in the cladding is slightly less, about 1.48. The index of refraction of air is approximately 1.0.

The fiber coating is colored using manufacturer's standard color codes to facilitate the identification of fiber. Like glass, optical fibers can be made completely from plastic. Optical fibers which are made from plastic are usually less expensive but have higher attenuation (loss) and limited application [7].

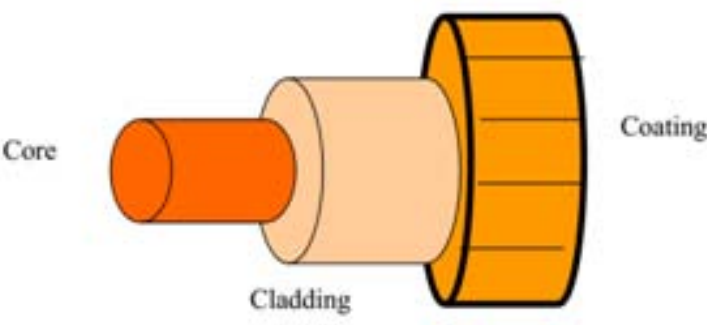

Fig (2): Optical fiber composition

A multimode fiber is a fiber that propagates more than one mode of light. The maximum number of modes of light (light ray path) that can exist in a fiber core can be estimated mathematically by the following expression [4].

$$
M=1+2 D\left(n_{1}^{2}+n_{2}^{2}\right)^{0.5} / \lambda
$$

Where $\mathrm{D}=$ core diameter

$\mathrm{n}_{1}=$ the core index of refraction

$\mathrm{n}_{2}=$ the cladding index of refraction

$\lambda=$ the wavelength of light

Fiber with large core diameter (greater than 10 micrometers) may be analyzed by geometrical optics. Such fiber is called multimode fiber, from the electromagnetic analysis. In a stepindex multimode fiber, rays of light are guided along the fiber core by total internal reflection. Rays that meet the core cladding boundary at a high angle (measured relative to a line normal to the boundary), greater than the critical angle for this boundary, are completely reflected. The critical angle (minimum angle for total internal reflection) is determined by the difference in index of refraction between the core and cladding materials. Rays that meet the boundary at a low angle are refracted from the core in to the cladding, and do not convey light and hence information along the fiber. The critical angle determines the acceptance angle of the fiber, often reported as a numerical aperture. A high numerical

Volume 5 Issue 2, February 2016 


\section{International Journal of Science and Research (IJSR) \\ ISSN (Online): 2319-7064 \\ Index Copernicus Value (2013): 6.14 | Impact Factor (2014): 5.611}

aperture allows light to propagate down the fiber in rays both close to the axis and at various angles, allowing efficient coupling of light into the fiber .However, this high numerical aperture increases the amount of dispersion as rays at different angles have different path length and therefore take different times to traverse the fiber.In graded - index fiber, the index of refraction in the core decreases continuously between the axis and the cladding. This causes light rays to bend smoothly as they approach the cladding, rather than reflecting abruptly from the core-cladding boundary. The resulting curved paths reduce multi-path dispersion because high angle rays pass more through the lower-index periphery of the core, rather than the high-index center. The index profile is chosen to minimum the difference in axial propagation speed of the various rays in the fiber. This ideal index profile is very close to a parabolic relationship between the index and distance from the axis [8].

Fiber with a core diameter less than about ten times the wavelength of propagating light cannot be modeled using geometric optics. Instead, it must be analyzed as an electromagnetic structure, by solution of Maxwell's equations as reduced to the electromagnetic wave equation. The electromagnetic analysis may also be required to understand behaviors such as speckle that occur when coherent light propagates in multi-mode fiber. As an optical waveguide, the fiber supports one or more confined transverse modes by which light can propagate along the fiber. Fiber supporting only one mode is called single- mode or mono-mode fiber. The behavior of larger - core multi -mode fiber can also be modeled using the wave equation, which shows that such fiber supports more than one mode of propagation. The results of such modeling of multi-mode fiber approximately agree with the predictions of geometric optics, if the fiber core is large enough to support more than a few modes. The most common type of single-mode fiber has a core diameter of $8-10$ micrometers and is designed for use in the near infrared. The mode structure depends on the wavelength of the light used, so that this fiber actually supports a small number of additional modes at visible wavelengths. Multi-mode fiber, by comparison, is manufactured with core diameters as small as 50 micrometers and as large as hundreds of micrometers. The normalized frequency $(v)$ for this fiber should be less than the first zero of the Bessel function ( $\mathrm{J}_{0}$ ) approximately (2.405) [9].

\section{The Mathematical Model}

Let suppose the optical fiber is have length (L ) meter and a diameter the core $(\mathrm{R})$ micrometer, light ray is incident on the core surface at critical angle $(\alpha)$, see the fig.(3):

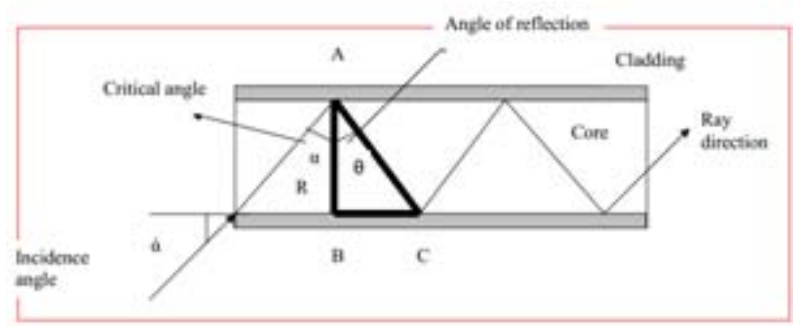

Fig (3): The cotal internal of reflection

$\because$ Critical angle $(\alpha)=$ Angle of Reflection $(\theta)$ (Snell's law) (2)

When see the triangle $(\mathrm{ABC})$ :

$$
\cos (\theta)=A B / A C
$$

But the diameter a core

$$
\begin{gathered}
R=A B \\
\therefore \cos (\theta)=R / A C \\
A C=R / \cos (\theta) \\
\because \sin (\theta)=B C / A C
\end{gathered}
$$

By using eq. (6) and substitution in eq. ( 7 ) to obtain on :

$$
\begin{gathered}
\sin (\theta)=B C /(R / \cos (\theta)) \\
\therefore B C=R \sin (\theta) / \cos (\theta) \\
B C=R \tan (\theta)
\end{gathered}
$$

When the number of total internal reflection $\left(\mathrm{N}_{\mathrm{r}}\right)$ is equal relative between the optical fiber length (L) and the piece of straight line which opposite the angle of reflection (BC) .

$$
\begin{gathered}
N_{r}=L / B C \\
N_{r}=L / R \tan (\theta)
\end{gathered}
$$

The last equation is correct when the optical fiber is straight line not suffer any bent.

\section{Results and Discussion}

\subsection{Optical fiber length (OFL)}

For study this point must be fixed the diameter a core ( R ) and the critical angle ,therefore the angle of reflection is limited from the Snell's law. The study is resulted the optical fiber length (OFL) is direct proportionality with the number total internal reflection angles $(\mathrm{Nr})$, see the fig( 4$)$. 


\section{International Journal of Science and Research (IJSR) \\ ISSN (Online): 2319-7064}

Index Copernicus Value (2013): 6.14 | Impact Factor (2014): 5.611

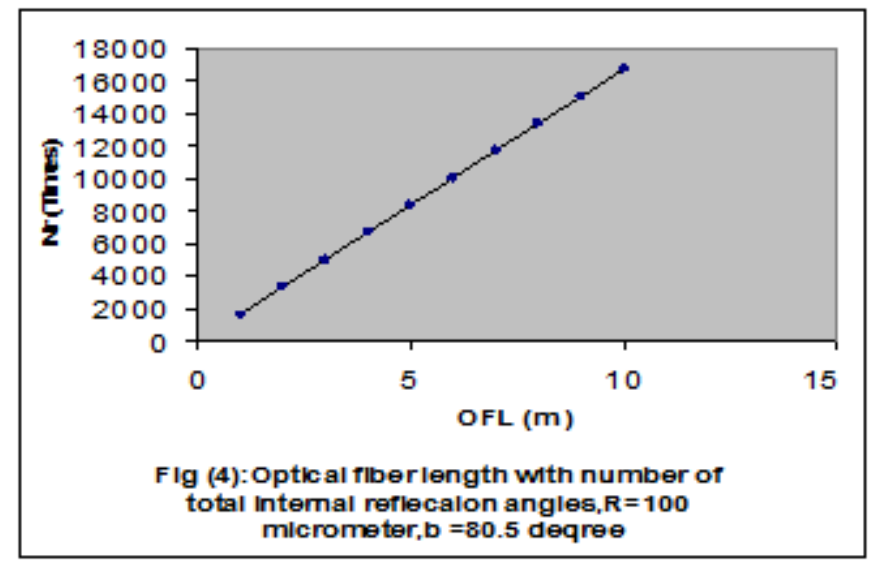

\subsection{Diameter of Core $(\mathbf{R})$}

To find the relationship between the number of total internal reflection angles $(\mathrm{Nr}$ ) and the diameter a core ( $\mathrm{R}$ ) must be fixed the optical fiber length ( OFL) and the angle of reflection $(\theta)$. This study is resulted the number of total internal reflection angles is indirect proportionality with diameter of core, see the fig. (5):

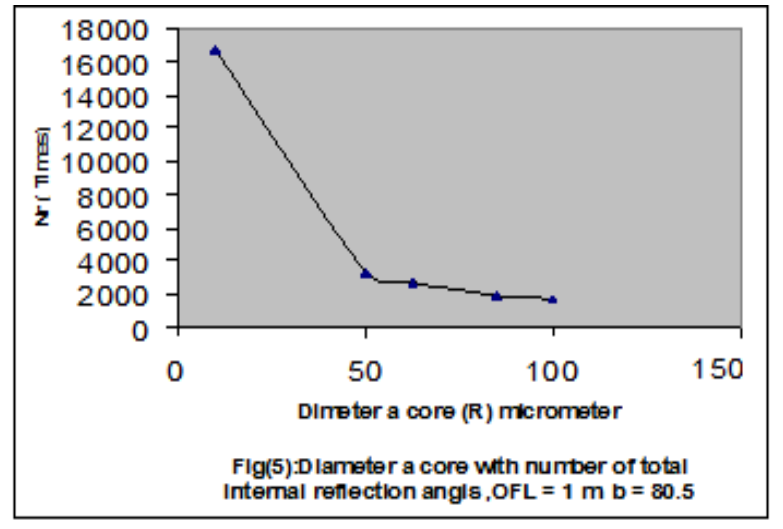

\subsection{Angle of reflection $(\theta)$}

To limit the angle of reflection must be to find the critical angle, therefore to find the relative index of refraction for many materials are contains the optical fiber, and use this equation:

Critical angle $=\operatorname{arcsine}\left(\mathrm{n}_{2} / \mathrm{n}_{1}\right)$

Where $\mathrm{n}_{1}=$ the index of refraction the first material

$\mathrm{n}_{2}=$ the index of refraction the second material

After ttohe find the critical angle can be obtain the angle of reflection according the Snell's law. When the study this point well fixed the optical fiber length (OFL) and the diameter of core (R). This study is produce the number of total internal reflection angles is indirect proportionality with tan function for angle of reflection see the fig (6):

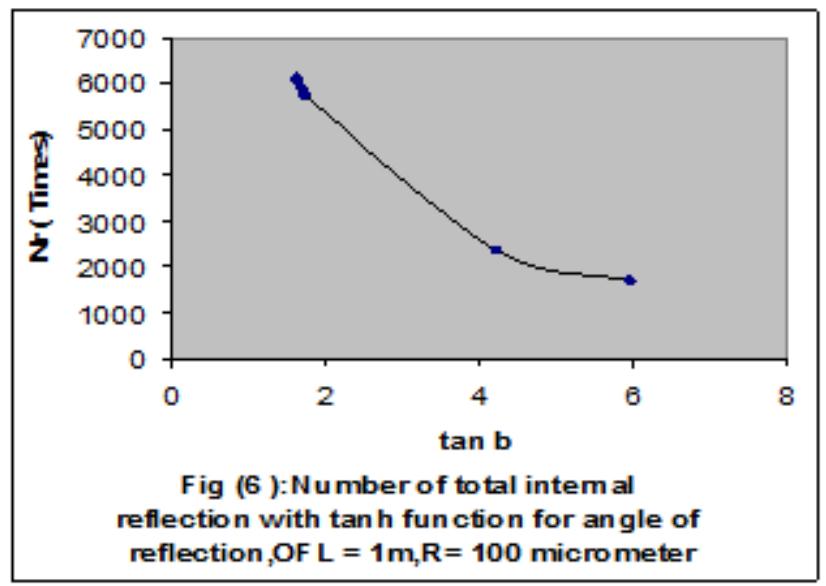

\section{Conclusions}

The results above reported can be indicating that the mathematical model is satisfactory, the results show that the number of total internal reflection angles is depend on the optical fiber characteristics ( length the fiber, diameter a core and index of refraction for both a core and cladding ). The study proved is the number of total internal reflection angles is direct proportionality with the optical fiber length while is indirect proportionality with the diameter of core and the tan function for angle of reflection. When the number of the total internal reflection is decreases, therefore this case is caused decreases in optical power such as Fresnel reflection. The mathematical model is useful at design the optical fiber.

\section{References}

[1] Jim Hayes, "The Story of Fiber Optics", Oxford University Press, New YorK,(1999).

[2] Jeff Hecht, "A Short History of Fiber Optics “,Report from Fiber Optics Technician's,Delmar Publishers, Albany, New York, (2010).

[3] Bates, Regis, J., "Optical”, Report of Wikipedia,(2001).

[4] Bob Chomycz, "FIBER OPTIC INSTALLER'S FIELD MANUAL", McGraw. Hill, ( 2000).

[5] Gambling, W.A., "The rise and rise of optical fibers "IEEE J.Sel.Top.Quantum Electron.6 (6), 1084,(2000).

[6] Mollers,D.Jager, Gaudino,R. ,Nocivelli, A., kragl, H., Ziemann,O., Weber, N., Koonen,T., Lezzi,C., Bluschke, A., Randel,S., "Plastic Optical Fiber Technology for Reliable Home Network", IEEE,Communication Magazine, Optical Communication Series, Vol. 47 , No. 8, pp.58-68,(2009).

[7] Craig Freudenrich, "How Fiber Optics Work", Report of Get Stuff,(2010).

[8] Ajeet Kumar, Vipul Rastogi “ Design and analysis of multilayer cladding large- mode-area optical fiber", J.Opt. A: Pure Appl.Opt.10 015303(pp. 6),(2008).

[9] Alexeyev, C.N., Alexeyev, A.N. and Yavorsky, M.A., "Effect of one-axis anisotropy on the propagation of optical vortices in rotating optical fibers", J.Opt. A: Pure Appl. Opt. 7 63-72, ( 2005). 